\title{
Quinolinic acid toxicity on oligodendroglial cells: relevance for multiple sclerosis and therapeutic strategies
}

\author{
Gayathri Sundaram ${ }^{1,2}$, Bruce J Brew ${ }^{1,3}$, Simon P Jones ${ }^{1}$, Seray Adams ${ }^{2,4}$, Chai K Lim, ${ }^{1,2}$ and Gilles J Guillemin ${ }^{1,2,4^{*}}$
}

\begin{abstract}
The excitotoxin quinolinic acid, a by-product of the kynurenine pathway, is known to be involved in several neurological diseases including multiple sclerosis (MS). Quinolinic acid levels are elevated in experimental autoimmune encephalomyelitis rodents, the widely used animal model of MS. Our group has also found pathophysiological concentrations of quinolinic acid in MS patients. This led us to investigate the effect of quinolinic acid on oligodendrocytes; the main cell type targeted by the autoimmune response in MS. We have examined the kynurenine pathway (KP) profile of two oligodendrocyte cell lines and show that these cells have a limited threshold to catabolize exogenous quinolinic acid. We further propose and demonstrate two strategies to limit quinolinic acid gliotoxicity: 1) by neutralizing quinolinic acid's effects with anti-quinolinic acid monoclonal antibodies and 2) directly inhibiting quinolinic acid production from activated monocytic cells using specific KP enzyme inhibitors. The outcome of this study provides a new insight into therapeutic strategies for limiting quinolinic acid-induced neurodegeneration, especially in neurological disorders that target oligodendrocytes, such as MS.
\end{abstract}

Keywords: Multiple sclerosis, Oligodendrocyte, Quinolinic acid, Excitotoxicity, Neurodegeneration, Neuroinflammation

\section{Introduction}

Quinolinic acid (QUIN) is a downstream metabolite produced through the kynurenine pathway (KP) of tryptophan metabolism [1,2]. In physiological conditions, QUIN is present in nanomolar concentrations and used as substrate by cells to synthesize the essential co-factor nicotinamide adenine dinucleotide $\left(\mathrm{NAD}^{+}\right)$. However, during neuroinflammation, the KP can be chronically or acutely activated through the induction of one of its initial enzymes, indoleamine 2,3-dioxygenase (IDO-1). QUIN is then produced in excess and can kill brain cells including neurons, astrocytes and oligodendrocytes by at least six different mechanisms [2,3]. QUIN has been implicated in several neurological diseases, including multiple sclerosis (MS) $[4,5]$. The involvement of the KP in neurological diseases is complex, as it revolves around the metabolic

\footnotetext{
* Correspondence: gilles.guillemin@mq.edu.au

${ }^{1}$ Applied Neurosciences Program, Peter Duncan Neurosciences Research Unit, St Vincent's Centre for Applied Medical Research, Sydney, Australia ${ }^{2}$ School of Medical Sciences, Faculty of Medicine, University of New South Wales, Sydney, Australia

Full list of author information is available at the end of the article
}

balance between excessive production of neurotoxic metabolites, such as QUIN, and neuroprotective compounds, such as kynurenic acid (KYNA) [6,7].

At the cellular level, QUIN is cytotoxic for most of the brain cell types in vitro at micromolar concentrations. Cammer [8,9] showed that exposure to $1 \mathrm{mM}$ of QUIN induces cell death in rat oligodendrocytes $[8,9]$. Similar toxic effects are also observed in primary human astrocytes and neurons at pathophysiological concentrations of $150 \mathrm{nM} \mathrm{[10],} \mathrm{and} \mathrm{more} \mathrm{recently} \mathrm{in} \mathrm{motor} \mathrm{neurons} \mathrm{at}$ concentrations of $100 \mathrm{nM}$ [11]. Furthermore, this effect can be abolished by using antagonists of the N-methylD-aspartate (NMDA) receptor - such as memantine, MK801 and AP-V - implying excitotoxicity as the main mechanism inducing cell death $[10,11]$. Current evidence suggests only monocytic lineage cells have the ability to produce QUIN $[12,13]$. Brain cell types, including neurons, astrocytes, pericytes and endothelial cells are likely to uptake QUIN and catabolize it [14-17].

The function of the KP in oligodendrocytes remains to be investigated, although an earlier study demonstrated that IDO-1 and tryptophan 2,3-dioxygenase (TDO-2) 
are not expressed in human primary oligodendrocytes [5]. This potentially has strong implications for MS pathology. The lack of these two KP regulatory enzymes in oligodendrocytes is associated with a higher cell susceptibility to allogenic T-cell challenge, since IDO-1 plays a crucial role in immune regulation - particularly in suppressing $\mathrm{T}$ cell proliferation [18]. The KP profile has been shown to be altered in both MS patients and in experimental autoimmune encephalitis (EAE) mouse models [19-21]. Rejdak et al. [19] reported that the concentration of the neuroprotective KYNA in the cerebrospinal fluid (CSF) of MS patients is decreased during the remission stage. This indicates that the KP stays inclined towards neurodegeneration long after inflammation. We hypothesise that in MS the sustained activation of the $\mathrm{KP}$ in the CNS leads to an unbalanced production favoring neurotoxic over neuroprotective intermediates.

This study investigates the profile of the KP in oligodendrocytes and characterizes to what extent KP metabolism changes during immune challenge. We have also identified novel approaches to protect oligodendrocytes against pathophysiological concentrations of QUIN. The outcomes of this study could have significant implications for potential therapeutic strategies in MS and other neurodegenerative diseases that involve KP dysregulation with overproduction of QUIN.

\section{Material and methods}

\section{Cell cultures}

Mouse oligodendroglial cell lines (N19 and N20.1) were kindly provided by Dr. A T Campagnoni (University of California, Los Angeles, CA, USA). These cells were conditionally immortalized oligodendrocytes isolated from normal and shiverer primary mouse brain cultures through the use of the retroviral vector pZIPSVtsA58 encoding immortalizing thermolabile simian virus 40 large $\mathrm{T}$ antigen that allows for clonal selection by conferring neomycin (G418) resistance [22]. N19 and N20.1 were chosen for their differences in developmental maturity along the oligodendrocyte lineage. N19 represent oligodendroglial cells with a relatively immature or precursor phenotype, whereas N20.1 represent a more mature phenotype. Oligodendroglial cells were cultured in accordance with the previously published method [22,23]. Briefly, cells were seeded and grown in flask coated with poly-L-lysine to confluence at $34^{\circ} \mathrm{C}$, the permissive temperature, in DMEM/F12; Invitrogen, Melbourne, Australia) supplemented with $10 \% \mathrm{FBS}, 100 \mu \mathrm{g} / \mathrm{ml}$ of gentamicin, $100 \mu \mathrm{g} /$ $\mathrm{ml}$ of G418 (Invitrogen, Melbourne, Australia) and then shifted to $39^{\circ} \mathrm{C}$, the non-permissive temperature that leads to 'differentiated' state for 72 hours. In all the experiments, during incubation at $39^{\circ} \mathrm{C}$, the medium was replaced by fresh DMEM/F12 with 1\% FBS and maintained for 7 days before treatments.
The BV2 cell line was kindly donated by Dr. T Kuffner (St Vincent's Center for Applied Medical Research, Sydney, Australia). The culture method for the BV2 murine microglial cells was adapted from Laurenzi and Arcuri et al. [24]. Briefly, BV2 cells were maintained in DMEM supplemented with 10\% FBS, Glutamax and antibiotic-anti-mycotic solution. The mouse macrophage cell line RAW264.7 was kindly donated by Prof. Nicholas Hunt (University of Sydney). The RAW264.7 cells were cultured based on the method adapted from Watts and Hunt et al. [25].

The study conditions were: (1) unstimulated; (2) IFN- $\gamma$ stimulated oligodendroglial cell lines (N19 and N20.1); and (3) IFN- $\gamma$-treated macrophage cell line, RAW 264.7 as a positive control, since macrophages are known to express all the KP enzymes [14]. The study design included 3 time points: 24,48 and 72 hours based on previous similar studies showing peak mRNA expression of KP enzymes at 24 hours, whilst production of KP metabolites peaks at 72 hours post-treatment (data not shown) $[15,16]$.

\section{Characterization of KP enzymes by quantitative RT-PCR}

The qRT-PCR protocol used has been previously described [26]. The primer sequences used to detect mouse KP enzymes are summarized in Table 1 . Primers were obtained from several sources: designed by Dr. Fabrice Magnino (PCR/qPCR specialist Integrated Sciences Pty Limited), a gift from collaborating researchers, Primer Bank (http://pga.mgh.harvard.edu/primerbank/) or designed by using Primer BLAST (web-based NCBI primer designing tool: http://www.ncbi.nlm.nih.gov/tools/primerblast/) or Primer3 designing program (http://primer3. sourceforge.net/). RPL13 was used as the endogenous reference gene. Data are presented as fold differences in gene expression normalized to RPL13 and relative to the untreated control. Differences in the relative expression of each gene were analyzed using Student's $t$-test. Statistical significance was accepted at $P<0.05$. Reported values are mean \pm SE of triplicate samples.

\section{Immunocytochemistry}

To characterize the KP in both N19 and N20.1 cell lines, cells were cultured onto slide flasks (Nunc, Rochester, NY, USA) at $34^{\circ} \mathrm{C}$ and when $70 \%$ confluence was reached they were incubated at $39^{\circ} \mathrm{C}$ for 72 hours. After 3 days, the cells were either left untreated or treated with $100 \mathrm{IU} / \mathrm{ml}$ of IFN- $\gamma$ for 24 hours. The cells were then stained for indoleamine 2,3-dioxygenase 1 (IDO-1) (Biolegend, San Diego, CA, USA), tryptophan 2,3-dioxygenase (TDO-2; generous gift from Dr. CL Miller John Hopkins University, Baltimore, MD, USA), kynurenine 3-monooxygenase (Abcam, Cambridge, UK), QUIN (Millipore, Bedford, MA, USA), picolinic acid (PIC; Abcam, Cambridge, UK), 2',3'cyclic nucleotide 3'-phosphodiesterase (CNP; Millipore, 
Table 1 Primer sequences for qRT-PCR

\begin{tabular}{|c|c|c|c|c|}
\hline \multirow{2}{*}{$\begin{array}{l}\text { Gene } \\
\text { Indoleamine 2,3-dioxygenase }\end{array}$} & \multirow{2}{*}{$\frac{\mathrm{ID}}{\mathrm{IDO1}}$} & \multirow{2}{*}{$\begin{array}{l}\text { Accession number } \\
\text { NM_008324 }\end{array}$} & \multicolumn{2}{|c|}{ Primer sequence } \\
\hline & & & FWD & TGT GAA TGG TCT GGT CTC \\
\hline & & & REV & CTG TGC CCT GAT AGA AGT \\
\hline \multirow[t]{2}{*}{ Tryptophan 2,3-dioxygenase } & TDO2 & BC018390.1 & FWD & TGC TCA AGG TGA TAG CTC GGA \\
\hline & & & REV & AGG AGC TTG AAG ATG ACC ACC A \\
\hline \multirow[t]{2}{*}{ Kynurenine aminotransferase 1} & KAT1 & NM_172404 & FWD & GCT TाT CAG CAG GCT ACC AC \\
\hline & & & REV & CCA CTG TCA CCA GCA CAT TC \\
\hline \multirow[t]{2}{*}{ Kynurenine aminotransferase 2} & KAT2 & AF072376 & FWD & GAA CTT CTG TCC TGG CTA A \\
\hline & & & REV & CTT GAT TGG GTG GGT AGT \\
\hline \multirow[t]{2}{*}{ Kynureninase } & KYNU & NM_027552 & FWD & GAG CAG AGG AGC GTG GCT GC \\
\hline & & & REV & GAA CAG GGG CCA CGC GGA TG \\
\hline \multirow[t]{2}{*}{ 3-hydroxyanthranilate 3,4-dioxygenase } & $3 \mathrm{HAO}$ & NM_025325 & FWD & TTC AGC CTC ATT GCA TCT \\
\hline & & & REV & GAC AGT GTA GGG CTA TGG \\
\hline \multirow[t]{2}{*}{ Kynurenine 3-monoxygenase } & $\mathrm{KMO}$ & NM_133809.1 & FWD & GGT CGC CTT CAC CAG AAT AA \\
\hline & & & REV & ATC CAG GCA GGT CTT CTC AA \\
\hline \multirow[t]{2}{*}{ Aminocarboxymuconate semialdehyde decarboxylase } & ACMSD & NM_001033041 & FWD & GAA TAA ATG CTG ACC CAA CA \\
\hline & & & REV & TTC ATC CAT CCT TCC AGA C \\
\hline \multirow[t]{2}{*}{ Quinolinate phosphoribosyl transferase } & QPRT & NM_133686.1 & FWD & CTG CTC CAA GTC ACC ATG \\
\hline & & & REV & CAG AAC CCC AGG AGA TाT \\
\hline \multirow[t]{2}{*}{ Ribosomal protein L13 } & RPL13 & NM_016738.5 & FWD & GAG GTC GGG TGG AAG TAC CA \\
\hline & & & REV & TGC ATC TTG GCC TाT TCC Tा \\
\hline
\end{tabular}

Bedford, MA, USA) and myelin basic protein (MBP; Abcam, Cambridge, UK) according to previously described protocol $[15,16]$. The antibodies are summarized in Tables 2 and 3. Untreated cells were stained and used as experimental control for comparison. Experiments were performed in triplicate and independently validated with three biological replicates.

\section{Quantification of KP metabolites}

Tryptophan, kynurenine, and kynurenic acid were measured using high performance liquid chromatography (HPLC) while picolinic acid and QUIN were concurrently measured using gas chromatography/mass spectrometry
(GC/MS) as previously described [11]. Results of tryptophan and kynurenine were presented as kynurenine/tryptophan $(\mathrm{K} / \mathrm{T})$ ratio, a reliable measure of $\mathrm{KP}$ activation. Production or degradation of the KP metabolites was calculated by subtracting the values of post- and pre-treatment concentrations of the KP metabolites present in the culture medium.

\section{QUIN toxicity and cell protection assays}

QUIN toxicity and cell protection assays against QUIN were determined by calculating the percentage of cell survival rate in comparison to untreated controls using CytoTox $96^{\circ}$ Non-Radioactive cytotoxicity assay kit (Promega,

Table 2 Summary of primary antibodies

\begin{tabular}{|c|c|c|c|}
\hline Antibody & Dilution & Isotype & Company \\
\hline Myelin basic protein (MBP) & $1: 100$ & Monoclonal - lgG & Abcam \\
\hline 2',3'-cyclic nucleotide 3'-phosphodiesterase (CNP) & $1: 50$ & Monoclonal - lgG & Millipore \\
\hline Vimentin (VIM) & $1: 100$ & Monoclonal - lgG & Millipore \\
\hline Glial fibrillary acidic protein (GFAP) & $1: 750$ & Monoclonal - lgG & Dako \\
\hline Indoleamine 2,3-dioxygenase 1 (IDO1) & $1: 100$ & Monoclonal - lgG & Biolegend \\
\hline Tryptophan 2,3-dioxygenase (TDO) & $1: 100$ & Polyclonal & {$[27]$} \\
\hline Kynurenine 3-monooxygenase (KMO) & $1: 100$ & Polyclonal & Abcam \\
\hline Quinolinic acid (QUIN) & $1: 100$ & Monoclonal - lgG & Millipore \\
\hline Picolinic acid (PIC) & $1: 100$ & Monoclonal - lgG & Abcam \\
\hline
\end{tabular}


Table 3 Summary of secondary antibodies

\begin{tabular}{llll}
\hline Antigen & Dilution & Isotype & Company \\
\hline Alexa Fluor 488 (green) & $1: 250$ & Goat anti-mouse lgG & Invitrogen \\
Alexa Fluor 488 (green) & $1: 250$ & Goat anti-rabbit lgG & Invitrogen \\
Alexa Fluor 594 (red) & $1: 250$ & Goat anti-mouse lgG & Invitrogen \\
Alexa Fluor 594 (red) & $1: 250$ & Goat anti-rabbit lgG & Invitrogen \\
\hline
\end{tabular}

Madison, WI, USA). Experiments were divided into four parts:

A) QUIN toxicity on oligodendrocytes: to assess the QUIN toxicity, oligodendroglial cells were challenged with varying concentrations of exogenous QUIN up to $4 \mu \mathrm{M}$ over 3 time points $(24,48$ and 72 hours) and QUIN toxicity assessed. These data were used to determine the $\mathrm{LD}_{50}$ of QUIN toxicity and select the appropriate treatment period in subsequent QUIN antagonism experiments (Parts $C$ and $D)$.

B) QUIN uptake assays: to further demonstrate the uptake of QUIN in oligodendrocytes, cells were grown in slide flasks (Nunc, Rochester, NY, USA) and treated with $\mathrm{LD}_{50}$ dose described in Part $A$ for $0,30,60$ and 90 minutes using protocol adapted from [28]. QUIN uptake was then visualized using immunocytochemistry as described previously [11,28].

C) Neutralization of QUIN with an anti-QUIN monoclonal antibody (mAb): to fully assess the potential of neutralizing QUIN toxicity with an anti-QUIN mAb, we subjected the oligodendroglial cells to 2 different conditions: 1 . treated directly on oligodendroglial cell lines with exogenous QUIN followed by varying concentrations of QUIN-mAb with the following three conditions: (a) pre-treatment with QUIN (QUIN-PRE) for 72 hours at LD $_{50}$ concentration followed by the QUIN-mAb for 30 minutes; (b) pre-treatment with anti-QUIN mAb for 30 minutes followed by QUIN (QUIN-POST) at $\mathrm{LD}_{50}$ concentration for 72 hours and; (c) concomitant treatment with QUIN and the anti-QUIN mAb (QUIN + QUIN mAb) together for 72 hours. 2. treated with IFN- $\gamma$-treated BV2 cells supernatant (endogenous QUIN) on oligodendroglial cell lines followed by varying concentrations of QUIN mAb. Cell death was then determined by measuring lactate dehydrogenase (LDH) in the culture supernatant.

D) Inhibition of QUIN production with IDO-1 inhibitors: to imitate QUIN production during inflammation and immune activation, BV2 cells were stimulated with IFN- $\gamma$ for 24 hours to induce pathophysiological concentrations of QUIN production. Oligodendrocyte cell line cultures were then exposed to this QUIN-containing BV2 culture supernatant for 72 hours and assessed for QUIN toxicity. Further, the QUIN-producing BV2 cells were challenged with 4 specific IDO-1 inhibitors namely, 1-methyl-D-tryptophan (D-1MT), 1-methyl-L-tryptophan (L-1MT), 1-methyl-Dtryptophan (DL-1MT) and berberine (5,6-dihydro9,10-dimethoxybenzo[g]-1,3-benzodioxolo[5,6-a] quinolizinium) for 30 minutes to block QUIN production as a potential therapeutic strategy to alleviate QUIN toxicity during neuroinflammation.

\section{Statistical analysis}

Results are expressed as mean $\pm \mathrm{SE}$. Differences between treatment groups for RT-PCR, GC/MS and HPLC data were analyzed using Student's $t$-test. QUIN toxicity study results were compared using linear and multiple regression analysis. QUIN mAb treatment results were analyzed using the Mann-Whitney test. In all experiments a $P$-value $<0.05$ was considered significant.

\section{Results}

KP profiling in oligodendroglial cell lines N19 and N20.1

We found that both mice oligodendrocytic cell lines N19 and N20.1 have a functional KP. This is evident at gene, protein and metabolite level in N19 (Figure 1a-d) and N20.1 (Figure 1e-h). However, the profile of KP expression in N19 was significantly different from N20.1 (see Additional files 1 and 2). By comparing the levels of mRNA expression for the KP enzymes, after normalization with the endogenous reference gene RPL13, we showed that N20.1 had a much higher level of expression for TDO-2 $(P<0.001)$ than N19 (Figure 1a and e). This result was validated at protein level using immunocytochemistry (Figure $1 \mathrm{~b}$ and $\mathrm{f}$ ). Both cell lines showed increased mRNA expression for TDO2 when treated with pro-inflammatory cytokine IFN- $\gamma$. In N20.1 cells most KP enzymes were up-regulated by IFN- $\gamma$, except ACMSD (see Additional file 1). As expected, treatment with IFN- $\gamma$ led to an increased KP activation shown by the increased K/T ratio in both cell lines (Figure 1c and g). Interestingly this increase was due to an up-regulation of TDO-2 rather than IDO-1 gene expression in N19.

KAT-1 and KAT-2 expression in both N19 and N20.1 suggests that oligodendrocytes are capable of producing the neuroprotective metabolite KYNA. Indeed, KYNA was detected in the culture media of both oligodendrocytic cell lines following background subtraction (serum supplemented media). Although there was a significant increase in KAT-1 and KAT-2 gene expression in N20.1 cells (see Additional file 1) following IFN- $\gamma$ treatment, there was no significant difference in the levels of KYNA produced (Figure 1d and h). ACMSD activity was low in both cell lines (see Additional file 1) and no production of PIC was detected in culture media of the cell lines 

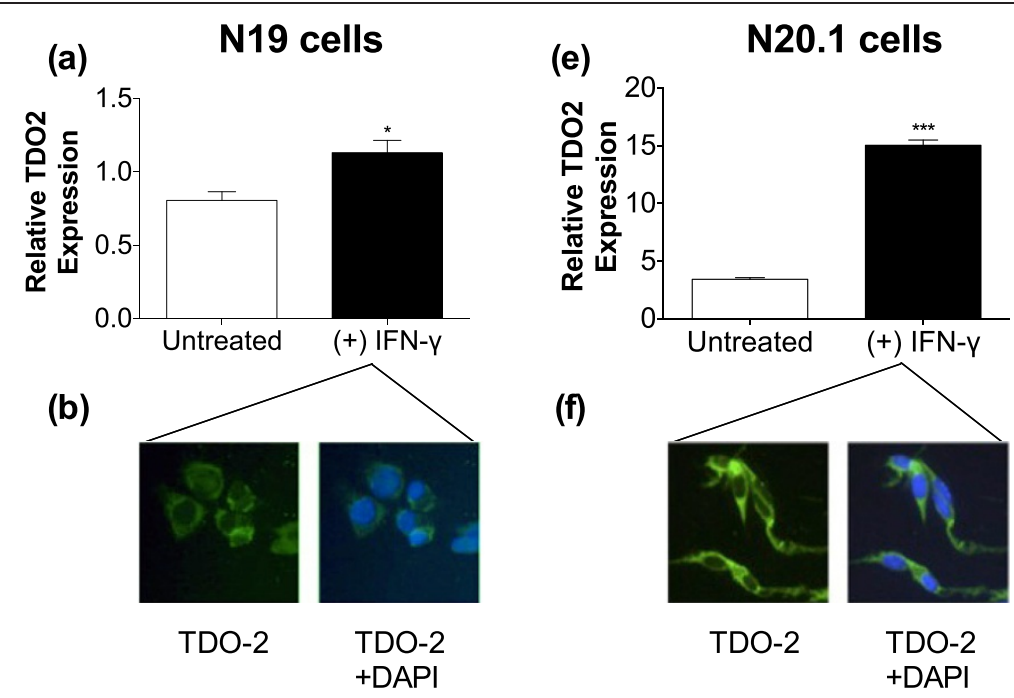

(c)

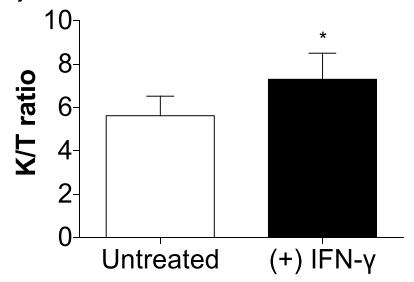

(d)

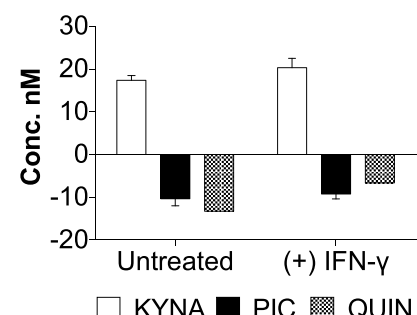

(g)

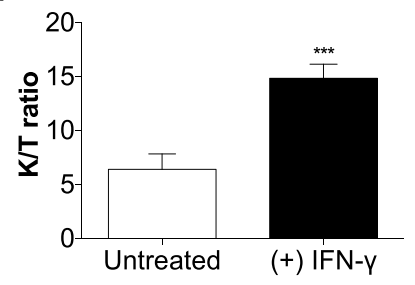

(h)

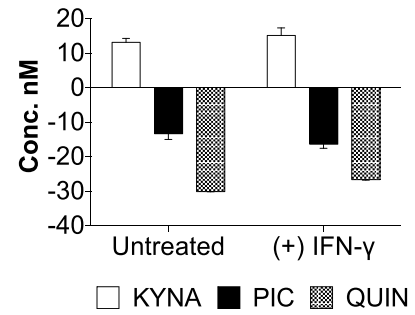

Figure 1 Characterization of the kynurenine pathway (KP) in N19 (a-e) and N20.1 (e-h) oligodendroglial cell lines. Levels of TDO-2 transcripts in (a) N19 and (e) N20.1 cells in differentiated state, with or without IFN- $\gamma$ treatment. N19 cells (b) and N20.1 cells (f) showed positive TDO-2 cytoplasmic staining after 24 hours IFN- $\gamma$ treatment. KP metabolic profile of oligodendroglial cell lines K/T ratio in N19 (c) and N20.1 (g) and kynurenic acid (KYNA), picolinic acid (PIC) and quinolinic acid (QUIN) profiles of N19 (d) and N20.1 (h) treated with or without IFN- $\gamma$. TDO-2, tryptophan 2,3-dioxygenase; K/ ratio, kynurenine and tryptophan ratio as a measure of IDO-1/TDO-2 activity; KYNA, kynurenic acid; PIC, picolinic acid; QUIN, quinolinic acid. Values are expressed as mean \pm SE. ${ }^{* *} P<0.001,{ }^{*} P<0.01$ and ${ }^{*} P<0.05$. Student's $t$-test.

(Figure $1 \mathrm{~d}$ and $\mathrm{h}$ ). Moreover, a negative value for PIC (N19: untreated: $-10.345 \mathrm{nM}$, IFN- $\gamma$ - treated: $-9.234 \mathrm{nM}$; N20.1: untreated: $-13.345 \mathrm{nM}$, IFN- $\gamma$-treated: -16.356 $\mathrm{nM}$ ) indicate post-culture concentrations were lower than that in pre-culture conditions. This suggests exogenous PIC, present in serum supplemented media, is taken up and/or catabolized by the oligodendrocytes. Low QUIN concentrations were also observed in the culture media of both cell types (Figure 1d and h). Along with the elevated activity of QPRT seen (see Additional file 1) this suggests an increased uptake and degradation of QUIN by oligodendroglial cells. To address this possibility we next investigated QUIN uptake by N19 and N20.1 cells.

Evidence of exogenous QUIN uptake in oligodendrocytes Using immunocytochemistry, we have demonstrated the capability of both oligodendrocyte cell lines to take up exogenous QUIN (Figure 2). This indicates QUIN is catabolized intracellularly in a time-dependent manner as fluorescence intensity was directly proportional to the uptake of QUIN. 


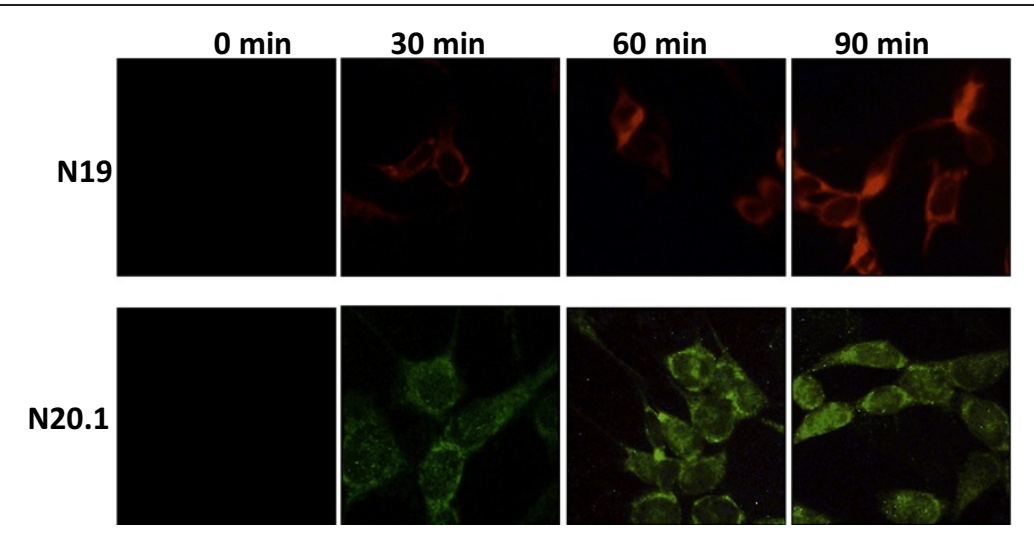

Figure 2 Quinolinic acid (QUIN) time-course study. N19 (top row) and N20.1 cells (bottom row) treated with specified concentration of QUIN for 0, 30, 60 and 90 minutes. Treated cells were analyzed by immunohistochemistry for QUIN uptake.

We then further assessed the cytotoxic threshold of such QUIN uptake on oligodendroglial cells. A standard curve, using commercial exogenous QUIN (Sigma-Aldrich, St Louis, MO, USA), was produced to assess the level of gliotoxicity by QUIN on N19 and N20.1 cells. This demonstrated a relatively similar level of tolerance in the two cell lines to QUIN (Figure 3a and b). There was a notable trend indicating dose-dependent QUIN toxicity on oligodendrocytes where the $\mathrm{LD}_{50}$ for $\mathrm{N} 19$ is $0.5 \mu \mathrm{M}$ and for $\mathrm{N} 20.1$ is $1 \mu \mathrm{M}$ for 72 hours. These $\mathrm{LD}_{50}$ values were chosen as the reference criteria for subsequent QUIN antagonism studies.

\section{Antagonism of QUIN toxicity with neutralizing anti-QUIN monoclonal antibody (mAb)}

Based on the 3 conditions illustrated in Figure $3 \mathrm{C}$ and the presence of $\mathrm{LD}_{50}$ concentrations of QUIN for 72 hours (determined in Figure $3 \mathrm{a}$ and $\mathrm{b}$ ), linear regression analysis showed that QUIN toxicity on N19 and N20.1 cell cultures was neutralized by QUIN mAb treatments (Figure $3 \mathrm{~d}$ and e). We found that treatment with anti-QUIN mAb diluted at $1 / 50(14 \mathrm{ng} / \mu \mathrm{L})$ led to complete protection against QUIN excitotoxicity. The pre-treatment with anti-QUIN mAb group was the least effective of the assessed treatments. Comparing the QUIN-PRE treatment groups between both cell lines, the effect of QUIN-induced cell death at $\mathrm{LD}_{50}$ in N20.1 was more reversible than N19. A validation study was performed to determine the concentration of anti-QUIN mAb used in the study was not toxic to the cells (data not shown).

In addition, we also demonstrated that this strategy worked on endogenous QUIN present in supernatants of IFN- $\gamma$-treated BV2 microglial cells containing $0.99 \pm$ $0.1 \mu \mathrm{M}$ of QUIN (data not shown). The glioprotection observed with the QUIN mAb treatment was found to be statistically different $(P<0.001)$ in all the treatment groups when compared to control cells for both the cell types (Figure 4). Among the treatment groups for both cell lines, anti-QUIN mAb at $1 / 100(7 \mathrm{ng} / \mu \mathrm{L})$ or higher concentration showed full protective effect from QUINinduced cell death.

\section{Inhibition of immune-induced QUIN toxicity with IDO inhibitors}

We tested 4 IDO-1 inhibitors including the natural compound berberine and 1-methyl-tryptophan (1MT) isoforms D, L and DL for their ability to block IDO-1 and so stop QUIN production by BV2 cells. We found that all four inhibitors were able to decrease QUIN induced toxicity in a dose dependent manner (Figure 5). Prior to study, IDO-1 inhibitors were tested for toxicity concentrations on BV2 cells and were well tolerated above $4 \mu \mathrm{M}$ (data not shown). At $1 \mu \mathrm{M}$, the 4 IDO- 1 inhibitors were able to bring the level of cytotoxicity under the $\mathrm{LD}_{50}$. At $4 \mu \mathrm{M}$, all the IDO- 1 inhibitors were able to completely abolish the QUIN-induced cell death in both cell lines. Comparing the percentage of cell death between the 2 cell lines, again we observed that N20.1 cells were slightly more sensitive to QUIN toxicity. A concentration of $0.5 \mu \mathrm{M}$ was needed to rescue N19 cells to $\mathrm{LD}_{50}$ whereas twice as much inhibitor was required to observe a similar effect in N20.1 cells $(P<0.001)$. Berberine was the less effective compound in protecting N19 cells whilst DL-1MT was the most effective in protecting the N20.1 cells during QUIN excitotoxicity.

\section{Discussion}

The KP profile has been extensively characterized in various human cell types including neurons, astrocytes, microglia, endothelial cells and several other immune cells [14-17]. However, the literature regarding the KP in oligodendrocytes is very limited [5]. More importantly the biological function of the KP in oligodendrocytes remains to be delineated. In this study, we showed that murine oligodendrocytic cell lines express all the KP 
(a)

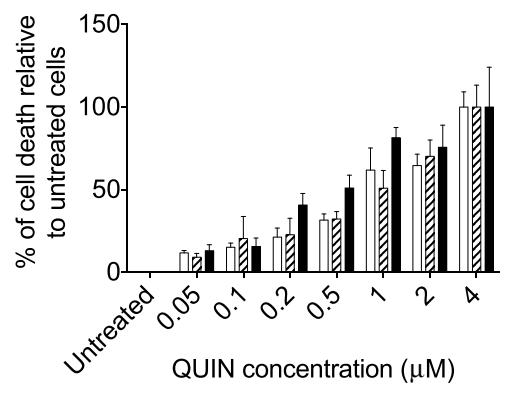

(b)

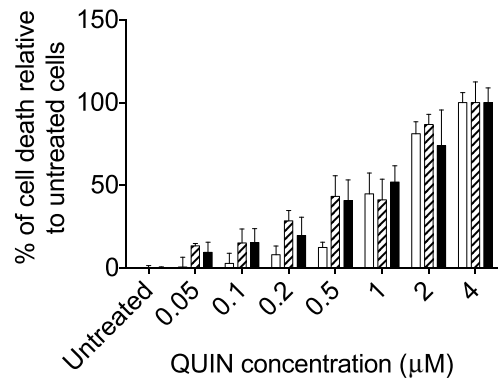

QUIN concentration $(\mu \mathrm{M})$

\section{$24 \mathrm{~h} \mathbb{Z} 48 \mathrm{~h} 72 \mathrm{~h}$}

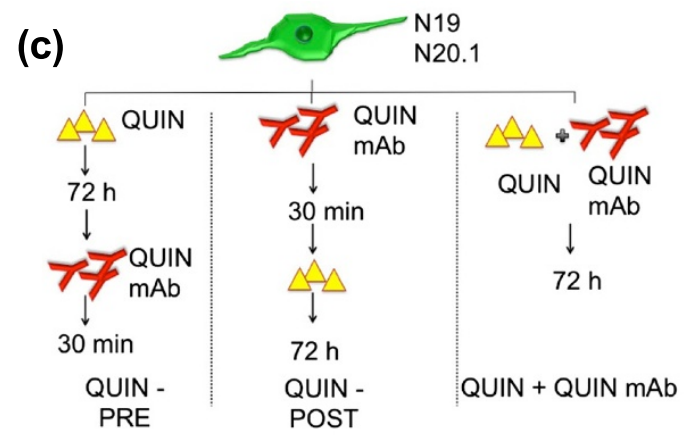

(d)

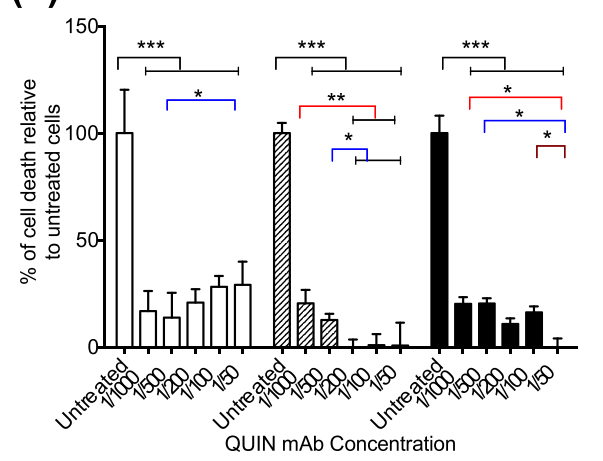

(e)

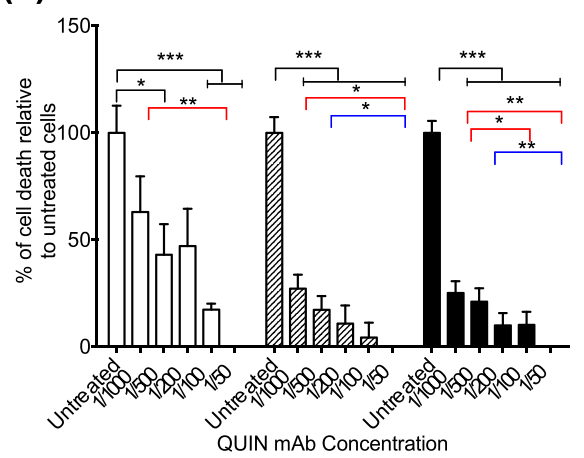

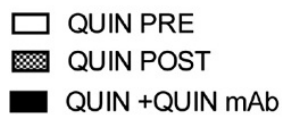

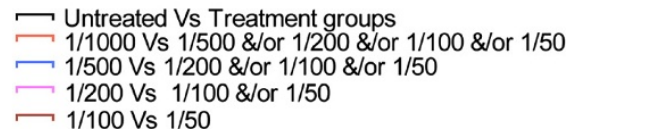

Figure 3 Neutralization of exogenous quinolinic acid (QUIN) with an anti-QUIN monoclonal antibody (mAb). The QUIN toxicity assay was investigated in (a) N19 and (b) N20.1 cells treated with various concentration of QUIN (0, 0.05, 0.1, 0.2, 0.5, 1, 2 and $4 \mu \mathrm{M})$ for 24 hours, 48 hours and 72 hours. Lactate dehydrogenase (LDH) activity in culture supernatant was determined. The results were analyzed using linear regression analysis. (c) The schematic diagram showed the treatment conditions of QUIN and QUIN mAb on oligodendroglial cells. The effect of QUIN mAb on QUIN toxicity was examined in (d) N19 and (e) N20.1 cells. The levels of LDH released by oligodendroglial cell lines after 72 hours of incubation with $0.5 \mu \mathrm{M}$ (N19) and $1 \mu \mathrm{M}$ (N20.1) of QUIN and varying concentrations of QUIN mAb (30 minutes). The study was performed in triplicate and the error bars indicate SE. ${ }^{* *} P<0.001$.

enzymes tested and have a fully functional KP unlike astrocytes, which lack the mid-stream enzyme KMO that converts kynurenine to 3-hydroxykynurenine [15]. However, these data conflict with our previous findings showing that human primary oligodendrocytes lack expression of both IDO-1 and TDO-2 [5]. These discrepancies are likely explained by the fact that KP expression: 1) varies between different species of the same cell type [29-31] and; 2 ) is significantly different between primary cells and their respective cell lines [5]. It is also possible that the 
(a)

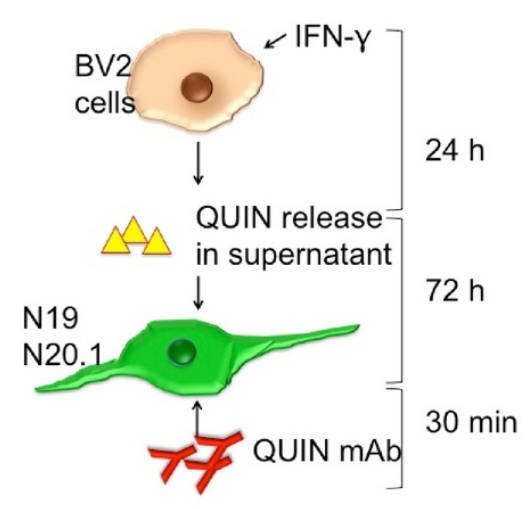

(b)

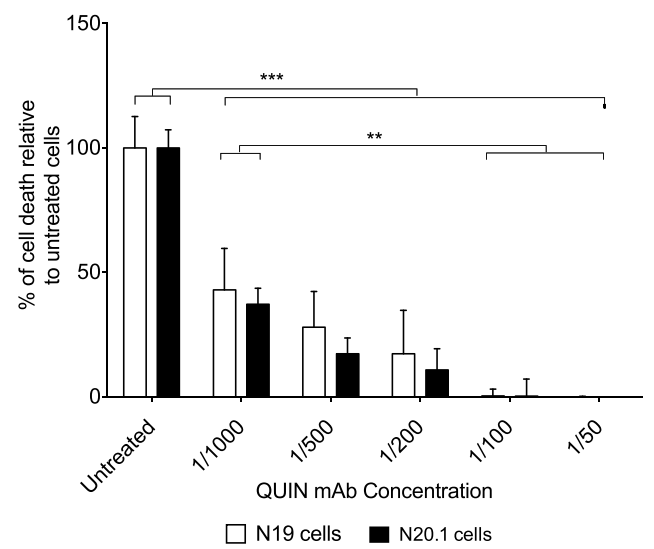

Figure 4 Neutralization of endogenous quinolinic acid (QUIN) with an anti-QUIN monoclonal antibody (mAb). (a) The schematic diagram represents the effect of QUIN mAb on IFN- $\gamma$-treated BV2 supernatant in N19 and N20.1 oligodendroglial cell lines. (b) represents the levels of lactate dehydrogenase (LDH) released by oligodendroglial cell lines N19 and N20.1, respectively after 72 hours incubation with IFN- $\gamma$-treated BV2 and varying concentrations of QUIN mAb. The study was performed in triplicate and the error bars indicate SE. ${ }^{* * *} P<0.001$.

conditional transfection of the oligodendrocytes with simian virus $40 \mathrm{~T}$ antigens may alter the KP expression [17]. Furthermore, in this study we showed that KP metabolism is different between two cell lines of the same cell type arising from the same species. N20.1 cells display a more active KP mRNA enzyme expression when compared to N19 (Figure 1). The degree of gene upregulation by IFN- $\gamma$ was also different: most of the KP enzymes were induced by IFN- $\gamma$ in N20.1 cells but only limited to TDO-2, KAT-2 and KYNU in N19 cells. The increase of K/T ratio as a result of TDO-2 up-regulation in these 2 mice oligodendrocytic cell lines was also reported by our group in the human oligodendrocytic cell line, MO3.13 [5]. It was also demonstrated in a recent study that TDO-2 is highly up-regulated in immortalized glial cell lines [32].
The expression of KATs and secretion of KYNA by both cell lines suggests that the oligodendrocytes are likely to have neuroprotective functions, especially during neuroinflammatory events. This is reflected by higher expression of KAT-II in the presence of IFN- $\gamma$ (see Additional file 1). This concurs with current literature pertaining to the mice model where KAT-2 is demonstrated to be the main enzyme associated with KYNA production in the brain [33]. Expression of QPRT corresponding with catabolic rather than anabolic activity of QUIN further supports the neuroprotective role of oligodendrocytes. In addition, this suggests that oligodendrocytes are capable of taking up exogenous QUIN present in the extracellular matrix. They could, therefore, be involved in the detoxification of pathophysiological concentrations of exogenous QUIN, secreted by

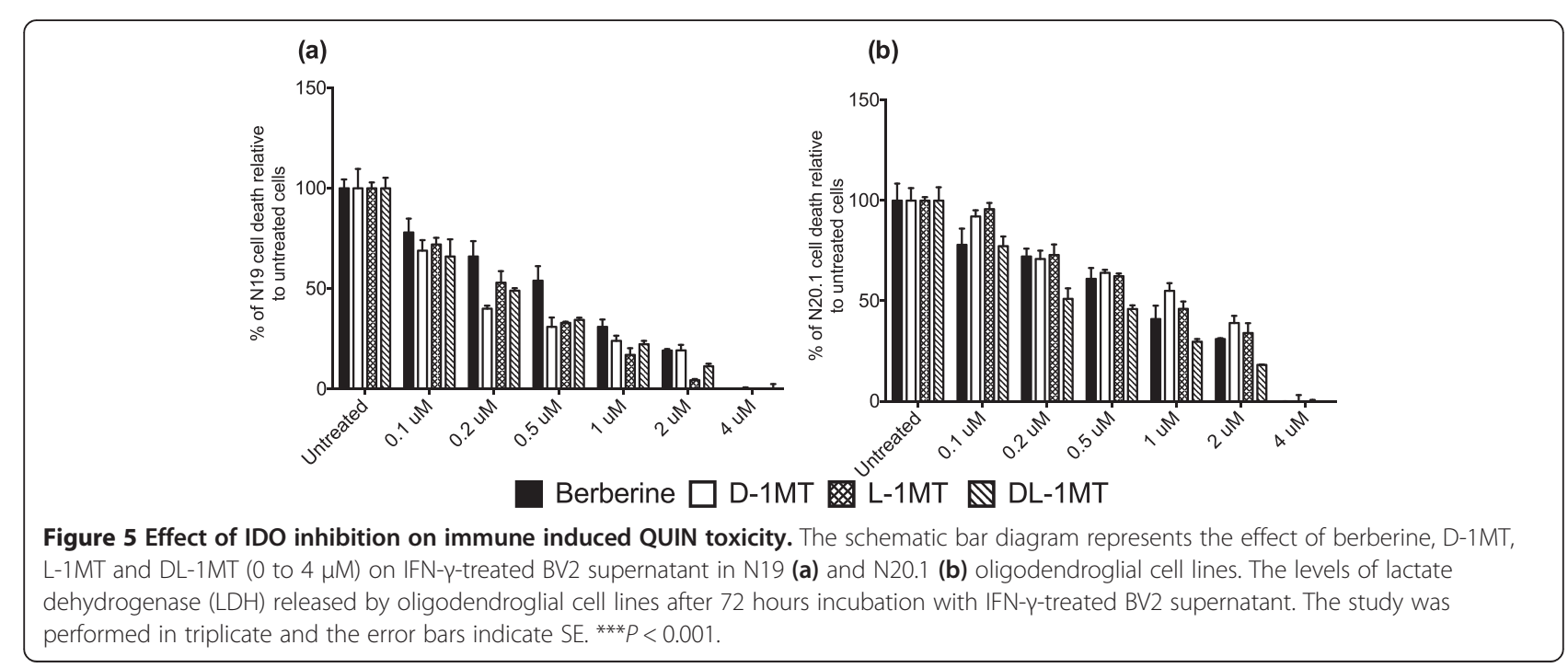


activated immune cells. Using immunocytostaining, we further showed a rapid uptake of QUIN by oligodendrocytes occurring within 30 minutes then gradually increasing with time (Figure 2). These results are in agreement with the KP metabolic profile found in human primary oligodendrocytes showing an uptake of exogenous QUIN from media and production of neuroprotective KYNA and PIC [5]. We previously showed that QUIN is cytotoxic to both primary human neurons and astrocytes at concentrations as low as $150 \mathrm{nM}$ and $350 \mathrm{nM}$ respectively [10].

As described above, N20.1 cells display a more active KP compared to N19 cells. This suggests that N20.1 cells are likely to have a higher tolerance to QUIN toxicity, that is a higher capacity to catabolize QUIN. Indeed, this was reflected by the $\mathrm{LD}_{50}$ concentration to QUIN toxicity in N20.1 cells with a difference 1-fold higher compared to N19 (Figure 3A). Surprisingly, this was not reflected in the PRE-QUIN treatment group where cells are pre-exposed to QUIN followed by treatment with anti-QUIN mAb (Figure 3D and E). We expected N20.1 cells to have a higher capacity to catabolize QUIN. However, the cell death assay revealed otherwise. It is important to note that the QUIN concentrations based on $\mathrm{LD}_{50}$ used in both cell lines are different. It may imply that QUIN at approximately $1 \mu \mathrm{M}$ concentration range may exert a more permanent and lasting excitotoxic effect. This is regardless of the cells capacity to take up and catabolize QUIN. An important aspect to further dissect is the short-term and long-term effects of QUIN toxicity on oligodendrocytes. This may have important implications for neurodegeneration and repair in situations where the KP is activated for sustained and prolonged periods of time, such as progressive MS. We have previously shown that low micro molar concentrations of QUIN could exert excitotoxicity chronically in human neurons over a period of 5 weeks [34]. Here, our data support that from a previous study by Cammer et al. [8] showing acute QUIN toxicity in primary rat oligodendrocytes at micro molar concentrations.

It is interesting to speculate that differences in susceptibility to QUIN-mediated toxicity observed between the two cell lines may also reflect their different maturation states and the associated expression of NMDA receptors. The relatively immature N19 cells, with a lower expression of NMDA receptors, experience reduced excitotoxicity in response to QUIN. Furthermore, it is also possible that there are differences in expression of QUIN transporter molecules and, therefore, varying levels of QUIN uptake possible between cell lines. The characterization of NMDA receptors and the capacity of the QUIN uptake transporter certainly warrant further investigation in oligodendrocyte development.

It is apparent that therapeutic strategies targeting QUIN could prevent excitotoxicity and ultimately significantly attenuate neurodegeneration. In this study, we demonstrated that neutralizing QUIN toxicity using an antiQUIN mAb could prevent oligodendrocyte cell death in vitro. There is still a limited number of studies targeting QUIN toxicity with a neutralizing antibody, despite extensive evidence showing QUIN as a key excitotoxin involved in several neuropathological diseases. This study is the first to propose the use of a mAb to neutralize in vitro QUIN toxicity. Based on our findings, the application of neutralizing QUIN with antibodies could be further developed into monoclonal antibody therapy for neurodegenerative diseases such as MS or amyotrophic lateral sclerosis [35]. Monoclonal antibody therapy has gained popularity over the years including the recently FDA approved natalizumab used in MS treatment [36,37].

As described above, chronic inflammatory responses can easily trigger cumulative production of pathophysiological concentrations of QUIN by activated monocytic cells such as infiltrating macrophages and microglia. This likely creates an environment in which neuronal cells are highly susceptible to excitotoxicity. Thus, we investigated further how manipulation of the KP during inflammation could affect the survival of oligodendrocytes towards QUIN-induced toxicity. We attempted to limit the production of QUIN from the BV2 microglial cell line by inhibiting IDO-1, since this enzyme is highly inducible by IFN- $\gamma$ and will be of pathophysiological relevance during inflammation. Our data show that the use of IDO-1 inhibitors (1-MT and berberine) were able to significantly decrease QUIN production by BV-2 cells and subsequently lead to complete abolishment of oligodendrocyte cell death at a concentration of $4 \mu \mathrm{M}$ (Figure 5). These results represent another relevant therapeutic strategy for MS using KP inhibitors. An earlier study using Ro 61-8048, a KMO inhibitor, on EAE rats showed a significant alleviation of disease progression [21]. In this same study the authors demonstrated that the advantage of $\mathrm{KMO}$ inhibition, in comparison with IDO-1, is that they could obtain both a decrease of QUIN synthesis and an increased production of the neuroprotective KYNA in the brain and spinal cord [21].

The use of IDO-1 inhibitors as a treatment for EAE and MS requires further investigation. The positive outcomes of this study are based on IDO-1 inhibition specifically in activated microglial cells. However, there is no IDO-1 inhibitor able to selectively target cell types. For example, inhibiting IDO-1 in astrocytes, the main producers of KYNA [15] would likely be deleterious. Furthermore, some studies have demonstrated that systemic inhibition of IDO-1 in EAE exacerbates the disease $[38,39]$. It is likely that the timing of intervention is important in this context: normal KP activation certainly suppresses aberrant immune responses and inhibiting this effect is unwanted in autoimmune disease. However, 
excessive KP activation produces neurotoxic metabolites, so preventing this dysregulated activation of the KP is clearly neuroprotective. Some KP metabolites have critical roles in the regulation of the T-cell activity responsible for autoimmune disorders [39-41]. This further supports the notion that the homeostatic activation of IDO-1 in EAE does have beneficial outcomes. However, its continued inhibition could also have significant negative effects in progressive disease.

It is technically challenging to deliver IDO- 1 inhibitors specifically to activated monocytic cells. However, cellspecific targeting might be possible using nanoparticles [42]. Another possibility will be to deliver multiple targets of KP modulators that can limit QUIN production whilst increasing KYNA and PIC. Platten et al. [43] demonstrated that tranilast (Rizaben), a synthetic analog of the KP intermediate 3-HAA, was able to fully abolish EAE by skewing the immunological profile from proinflammatory to immunosuppressive [43]. Combination therapy targeting KP enzymes and/or metabolites appears to be a promising therapeutic for EAE and MS but requires further investigation.

\section{Conclusions}

In conclusion, our data show that the oligodendrocytes express the major components of KP and, importantly, are capable of catabolizing exogenous QUIN. Therefore, they are likely to play a role in the detoxification of excessive QUIN associated with a chronic immune-activated KP in monocytic cells. More importantly, QUIN is toxic to oligodendrocytes at pathophysiological concentrations. Herein, we demonstrated two potential therapeutic strategies to overcome the toxicity of QUIN by directly neutralizing with anti-QUIN mAb and indirectly with $\mathrm{KP}$ inhibitors to limit QUIN production. This work presents novel therapeutic insights into excitotoxicity and neuroprotection for oligodendrocytes and other brain cell types.

\section{Main points}

- First characterization of the kynurenine pathway (KP) in oligodendrocytes

- Oligodendrocytes take up QUIN

- QUIN is toxic for oligodendrocytes

- Exogenous QUIN toxicity can be neutralized by using a monoclonal antibody against QUIN and specific KP inhibitors leading to increased oligodendrocyte survival

\section{Additional files}

Additional file 1: Expression of kynurenine pathway genes in oligodendroglial cells. Levels of transcripts in (a) N19 and (b) N20.1 oligodendroglial cell line in differentiated state, with or without IFN- $\gamma$ treatment. Dots represent mean value from four different experiments. The levels of transcripts from the genes encoding are IDO1 (indoleamine 2,3-dioxygenase), TDO2 (tryptophan 2,3-dioxygenase), KAT1 (kynurenine aminotransferase1), KAT2 (kynurenine aminotransferase2), KYNU (kynureninase), ACMSD (aminocarboxymuconate semialdehyde decarboxylase) and QPRT (quinolinate phosphoribosyl transferase). Horizontal line indicates median values. ${ }^{* *} P<0.001{ }^{* *} P<0.01$ and ${ }^{*} P<0.05$.

Additional file 2: Expression of kynurenine pathway protein in oligodendroglial cells. Characterization of the kynurenine pathway in (a) N19 and (b) N20.1 cells by immunohistochemistry. Cells showed positive cytoplasmic staining of TDO2 (N19 and N20.1) and KMO (N20.1 only) after 24 hours IFN- $\gamma$ treatment. IDO1, QUIN and PIC were absent in this

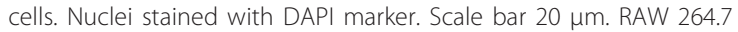
cells were used as control for positive-staining of IDO1, TDO2, KMO, QUIN and PIC.

\section{Abbreviations}

KP: kynurenine pathway; MS: multiple sclerosis; KYN: kynurenine; TRP: tryptophan; KYNA: kynurenic acid; PIC: picolinic acid; QUIN: quinolinic acid; mAb: monoclonal antibody; IDO-1: indoleamine 2,3-dioxygenase; TDO-2: tryptophan 2,3-dioxygenase; 1MT: 1-methyl-tryptophan.

\section{Competing interests}

The authors declare that they have no competing interests.

\section{Authors' information}

GS performed the experiments and acquired the data. GS, SA and CL analyzed the data. GS, CL and GG designed the study and interpreted the data. GS wrote the manuscript. BB, SJ, CL and GG critically revised the manuscript for important intellectual content. All authors read and approved the final manuscript.

CK and $G G$ are sharing senior authorship.

\section{Acknowledgement}

This work had been funded by the Australian Research Council, the National Health and Medical Research Council, St Vincent's Clinic Foundation and Peter Duncan Foundation. This study had also partly benefited from the Multiple Sclerosis Research Australia and Trish Foundation. The authors wish to acknowledge the Bioanalytical Mass Spectrometry Facility (BMSF) at UNSW for their kind support, especially Ms Sonia Bustamante for her technical expertise and kind advice with the GC/MS. The authors will also like to thanks Dr. Kuffner, Prof Nicholas Hunt and Dr. Campagnoni for their generous provision of cells.

\section{Author details}

${ }^{1}$ Applied Neurosciences Program, Peter Duncan Neurosciences Research Unit, St Vincent's Centre for Applied Medical Research, Sydney, Australia. ${ }^{2}$ School of Medical Sciences, Faculty of Medicine, University of New South Wales, Sydney, Australia. ${ }^{3}$ Department of Neurology, St Vincent's Hospital, Sydney, Australia. ${ }^{4}$ Neurodegenerative diseases Research Group, Australian School of Advanced Medicine, Faculty of Human Sciences, Macquarie University, Sydney, NSW 2109, Australia.

Received: 1 September 2014 Accepted: 17 November 2014 Published online: 13 December 2014

\section{References}

1. Bender DA, McCreanor GM: The preferred route of kynurenine metabolism in the rat. Biochim Biophys Acta 1982, 717:56-60.

2. Guillemin GJ: Quinolinic acid, the inescapable neurotoxin. FEBS J 2012, 279:1356-1365.

3. Stone TW: Endogenous neurotoxins from tryptophan. Toxicon 2001, 39:61-73.

4. Chen Y, Guillemin GJ: Kynurenine pathway metabolites in humans: disease and healthy States. Int J Tryptophan Res 2009, 2:1-19.

5. Lim CK, Smythe GA, Stocker R, Brew BJ, Guillemin GJ: Characterization of the kynurenine pathway in human oligodendrocytes. In Proceedings of the Eleventh Triennial Meeting of International Study Group for Tryptophan Research: Tokyo. Edited by Takai K. 2007:213-217. 
6. Stone TW: Neuropharmacology of quinolinic and kynurenic acids. Pharmacol Rev 1993, 45:309-379.

7. Lekieffre D, Plotkine $M$, Allix M, Boulu RG: Kynurenic acid antagonizes hippocampal quinolinic acid neurotoxicity: behavioral and histological evaluation. Neurosci Lett 1990, 120:31-33.

8. Cammer W: Oligodendrocyte killing by quinolinic acid in vitro. Brain Res 2001, 896:157-160.

9. Cammer W: Protection of cultured oligodendrocytes against tumor necrosis factor-alpha by the antioxidants coenzyme $\mathrm{Q}(10)$ and $\mathrm{N}$-acetyl cysteine. Brain Res Bull 2002, 58:587-592.

10. Braidy N, Grant R, Adams S, Brew BJ, Guillemin GJ: Mechanism for quinolinic acid cytotoxicity in human astrocytes and neurons. Neurotox Res 2009, 16:77-86.

11. Chen Y, Brew BJ, Guillemin GJ: Characterization of the kynurenine pathway in NSC-34 cell line: implications for amyotrophic lateral sclerosis. J Neurochem 2011, 118:816-825.

12. Espey MG, Chernyshev ON, Reinhard JF Jr, Namboodiri MA, Colton CA Activated human microglia produce the excitotoxin quinolinic acid. Neuroreport 1997, 8:431-434.

13. Heyes MP, Achim CL, Wiley CA, Major EO, Saito K, Markey SP: Human microglia convert L-tryptophan into the neurotoxin quinolinic acid. Biochem J 1996, 320:595-597.

14. Guillemin GJ, Smith DG, Smythe GA, Armati PJ, Brew BJ: Expression of the kynurenine pathway enzymes in human microglia and macrophages. Adv Exp Med Biol 2003, 527:105-112.

15. Guillemin GJ, Kerr SJ, Smythe GA, Smith DG, Kapoor V, Armati PJ, Croitoru J, Brew BJ: Kynurenine pathway metabolism in human astrocytes: a paradox for neuronal protection. J Neurochem 2001, 78:842-853.

16. Guillemin GJ, Cullen KM, Lim CK, Smythe GA, Garner B, Kapoor V, Takikawa $\mathrm{O}$, Brew BJ: Characterization of the kynurenine pathway in human neurons. J Neurosci 2007, 27:12884-12892.

17. Owe-Young R, Webster NL, Mukhtar M, Pomerantz RJ, Smythe G, Walker D, Armati PJ, Crowe SM, Brew BJ: Kynurenine pathway metabolism in human blood-brain-barrier cells: implications for immune tolerance and neurotoxicity. J Neurochem 2008, 105:1346-1357.

18. Mellor AL, Munn DH: Tryptophan catabolism and regulation of adaptive immunity. J Immunol 2003, 170:5809-5813.

19. Rejdak K, Bartosik-Psujek H, Dobosz B, Kocki T, Grieb P, Giovannoni G, Turski WA, Stelmasiak Z: Decreased level of kynurenic acid in cerebrospinal fluid of relapsing-onset multiple sclerosis patients. Neurosci Lett 2002, 331:63-65.

20. Flanagan EM, Erickson JB, Viveros OH, Chang SY, Reinhard JF: Neurotoxin quinolinic acid is selectively elevated in spinal-cords of rats with experimental allergic encephalomyelitis. J Neurochem 1995, 64:1192-1196.

21. Chiarugi A, Cozzi A, Ballerini C, Massacesi L, Moroni F: Kynurenine 3-monooxygenase activity and neurotoxic kynurenine metabolites increase in the spinal cord of rats with experimental allergic encephalomyelitis. Neuroscience 2001, 102:687-695.

22. Verity AN, Bredesen D, Vonderscher C, Handley WW, Campagnoni AT: Expression of myelin protein genes and other myelin components in an oligodendrocytic cell line conditionally immortalized with a temperature-sensitive retrovirus. J Neurochem 1993, 60:577-587.

23. Foster LM, Phan T, Verity AN, Bredesen D, Campagnoni AT: Generation and analysis of normal and shiverer temperature-sensitive immortalized celllines exhibiting phenotypic characteristics of oligodendrocytes at several stages of differentiation. Dev Neurosci 1993, 15:100-109.

24. Laurenzi MA, Arcuri C, Rossi R, Marconi P, Bocchini V: Effects of microenvironment on morphology and function of the microglial cell line BV-2. Neurochem Res 2001, 26:1209-1216.

25. Watts AD, Hunt NH, Madigan MC, Chaudhri G: Soluble TNF-a receptors bind and neutralize over-expressed transmembrane TNF-a on macrophages, but do not inhibit its processing. J Leukoc Biol 1999, 66:1005-1013.

26. Lee MC, Ting KK, Adams S, Brew BJ, Chung R, Guillemin GJ: Characterisation of the expression of NMDA receptors in human astrocytes. PLoS One 2010, 5:e14123.

27. Miller CL, Llenos IC, Dulay JR, Barillo MM, Yolken RH, Weis S: Expression of the kynurenine pathway enzyme tryptophan 2,3-dioxygenase is increased in the frontal cortex of individuals with schizophrenia. Neurobiol Dis 2004, 15:618-629.

28. Rahman A, Ting K, Cullen KM, Braidy N, Brew BJ, Guillemin GJ: The excitotoxin quinolinic acid induces tau phosphorylation in human neurons. PLOS One 2009, 4:e6344.
29. Fujigaki S, Saito K, Takemura M, Fujii H, Wada H, Noma A, Seishima M: Species differences in L-tryptophan-kynurenine pathway metabolism: quantification of anthranilic acid and its related enzymes. Arch Biochem Biophys 1998, 358:329-335.

30. Heyes MP, Saito K, Chen CY, Proescholdt MG, Nowak TS, Li J, Beagles KE, Proescholdt MA, Zito MA, Kawai K, Markey SP: Species heterogeneity between gerbils and rats: quinolinate production by microglia and astrocytes and accumulations in response to ischemic brain injury and systemic immune activation. J Neurochem 1997, 69:1519-1529.

31. Croitoru-Lamoury J, Lamoury FM, Caristo M, Suzuki K, Walker D, Takikawa O, Taylor R, Brew BJ: Interferon-gamma regulates the proliferation and differentiation of mesenchymal stem cells via activation of indoleamine 2,3 dioxygenase (IDO). PLoS One 2011, 6:e14698.

32. Opitz CA, Litzenburger UM, Sahm F, Ott M, Tritschler I, Trump S, Schumacher T, Jestaedt L, Schrenk D, Weller M, Jugold M, Guillemin GJ, Miller CL, Lutz C, Radiwimmer B, Lehmann I, von Deimling A, Wick W, Platten M: An endogenous tumour-promoting ligand of the human aryl hydrocarbon receptor. Nature 2011, 478:197-203.

33. Alkondon M, Pereira EF, Eisenberg HM, Kajii Y, Schwarcz R, Albuquerque EX: Age dependency of inhibition of alpha7 nicotinic receptors and tonically active $\mathrm{N}$-methyl-D-aspartate receptors by endogenously produced kynurenic acid in the brain. J Pharmacol Exp Ther 2011, 337:572-582.

34. Kerr SJ, Armati PJ, Guillemin GJ, Brew BJ: Chronic exposure of human neurons to quinolinic acid results in neuronal changes consistent with AIDS dementia complex. AIDS 1998, 12:355-363.

35. Kang SH, Li Y, Fukaya M, Lorenzini I, Cleveland DW, Ostrow LW, Rothstein $J D$, Bergles DE: Degeneration and impaired regeneration of gray matter oligodendrocytes in amyotrophic lateral sclerosis. Nat Neurosci 2013, 16:571-579.

36. Kelley $B$ : Industrialization of $m A$ b production technology: the bioprocessing industry at a crossroads. mAbs 2009, 1:443-452

37. Ransohoff RM: Natalizumab for multiple sclerosis. N Engl J Med 2007, 356:2622-2629.

38. Kwidzinski E, Bunse J, Aktas O, Richter D, Mutlu L, Zipp F, Nitsch R, Bechmann I: Indolamine 2,3-dioxygenase is expressed in the CNS and down-regulates autoimmune inflammation. Faseb J 2005, 19:1347-1349.

39. Sakurai K, Zou JP, Tschetter JR, Ward JM, Shearer GM: Effect of indoleamine 2,3-dioxygenase on induction of experimental autoimmune encephalomyelitis. J Neuroimmunol 2002, 129:186-196.

40. Matysiak M, Stasiolek M, Orlowski W, Jurewicz A, Janczar S, Raine CS, Selmaj K: Stem cells ameliorate EAE via an indoleamine 2,3-dioxygenase (IDO) mechanism. J Neuroimmunol 2008, 193:12-23.

41. Yan Y, Zhang GX, Gran B, Fallarino F, Yu S, Li H, Cullimore ML, Rostami A, Xu $\mathrm{H}$ : IDO upregulates regulatory T cells via tryptophan catabolite and suppresses encephalitogenic $T$ cell responses in experimental autoimmune encephalomyelitis. J Immunol 2010, 185:5953-5961.

42. Weissleder R, Kelly K, Sun EY, Shtatland T, Josephson L: Cell-specific targeting of nanoparticles by multivalent attachment of small molecules. Nat Biotechnol 2005, 23:1418-1423.

43. Platten M, Ho PP, Youssef S, Fontoura P, Garren H, Hur EM, Gupta R, Lee LY, Kidd BA, Robinson WH, Sobel RA, Selley ML, Steinman L: Treatment of autoimmune neuroinflammation with a synthetic tryptophan metabolite. Science 2005, 310:850-855.

\section{Submit your next manuscript to BioMed Central and take full advantage of:}

- Convenient online submission

- Thorough peer review

- No space constraints or color figure charges

- Immediate publication on acceptance

- Inclusion in PubMed, CAS, Scopus and Google Scholar

- Research which is freely available for redistribution 